Article

\title{
An Incentive Based Dynamic Pricing in Smart Grid: A Customer's Perspective
}

\author{
Thamer Alquthami ${ }^{1} * \mathbb{D}$, Ahmad H. Milyani ${ }^{1}\left(\mathbb{D}\right.$, Muhammad Awais $^{2}$ and Muhammad B. Rasheed ${ }^{3,4, *(D)}$ \\ 1 Electrical and Computer Engineering Department, King Abdulaziz University, Jeddah 21589, Saudi Arabia; \\ ahmilyani@kau.edu.sa \\ 2 Department of Technology, The University of Lahore, Lahore 54000, Pakistan; m.awais.qureshi27@gmail.com \\ 3 Department of Electronics and Electrical Systems, The University of Lahore, Lahore 54000, Pakistan \\ 4 Computer Engineering Department, University of Alcalá, 28805 Alcalá de Henares, Spain \\ * Correspondence: tquthami@kau.edu.sa (T.A.); Muhammad.rasheed@uah.es (M.B.R.)
}

check for

updates

Citation: Alquthami, T.; Milyani, A.H.; Awais, M.; Rasheed, M.B. An Incentive Based Dynamic Pricing in Smart Grid: A Customer's

Perspective. Sustainability 2021, 13, 6066. https://doi.org/10.3390/ su13116066

Academic Editors: Sheraz Aslam,

Herodotos Herodotou and Nouman Ashraf

Received: 20 April 2021

Accepted: 21 May 2021

Published: 27 May 2021

Publisher's Note: MDPI stays neutral with regard to jurisdictional claims in published maps and institutional affiliations.

Copyright: (c) 2021 by the authors. Licensee MDPI, Basel, Switzerland. This article is an open access article distributed under the terms and conditions of the Creative Commons Attribution (CC BY) license (https:/ / creativecommons.org/licenses/by/ $4.0 /)$.

\begin{abstract}
Price based demand response is an important strategy to facilitate energy retailers and end-users to maintain a balance between demand and supply while providing the opportunity to end users to get monetary incentives. In this work, we consider real-time electricity pricing policy to further calculate the incentives in terms of reduced electricity price and cost. Initially, a mathematical model based on the backtracking technique is developed to calculate the load shifted and consumed in any time slot. Then, based on this, the electricity price is calculated for all types of users to estimate the incentives through load shifting profiles. To keep the load under the upper limit, the load is shifted in other time slots in such a way to facilitate end-users regarding social welfare. The user who is not interested in participating load shifting program will not get any benefit. Then the well behaved functional form optimization problem is solved by using a heuristic-based genetic algorithm (GA), wwhich converged within an insignificant amount of time with the best optimal results. Simulation results reflect that the users can obtain some real incentives by participating in the load scheduling process.
\end{abstract}

Keywords: demand side management; demand response; load scheduling; real time pricing; genetic algorithm; dynamic incentives

\section{Introduction and Background}

The smart grid (SG) is an emerging paradigm shift in power distribution systems that aims to improve itself using various information and communication technologies. It comprises various intelligent controlling and decision-making systems, which manage electricity generation, transmission and distribution through two-way communication mechanisms [1,2]. In addition, SG allows the integration of distributed and renewable generation facilities to cope with various uncertainties (i.e., energy deficits, blackouts, high peaks) that are caused by the energy demand variations and intermittent nature of renewables [3], which helps minimize carbon emissions. This is due to the fact that distributed renewable energy resources can provide power during stand alone or independent/island mode to manage power demand with reduced emission. For this purpose, intelligent autonomous mechanism, blockchain technology and artificial intelligence are key technologies being widely adopted in recent decades. Furthermore, as distributed generation and renewable sources can play a key role in managing energy demand, distribution systems can use the flexibility of variable energy resources to improve the underlying capacity of low voltage distribution networks, which can also be referred to as "active distribution systems". Due to the developments in energy storage technologies, as well as the strong need to reduce transportation-related costs and emissions [3], the focus on electric vehicles has been increasing. Electric vehicles need to be recharged at charging stations. Thus, based on the aforementioned justifications, EVs integration has a threefold set of objectives: 
(i) it can be used for transportation purposes replacing the traditional vehicles due to a large amount of carbon emissions and abrupt fluctuations in fuel prices; (ii) EVs can also be used for the transmission of electricity; (iii) the integration of EVs can help with alleviating the high and rebound peaks majorly caused by high energy extracted during low pricing hours.

Recent advancement in communication and control technologies has envisioned the DR programs as an important tool for load management and scheduling. In [4], the authors used an opportunistic load scheduling technique, which is based on optimal stopping theory. Each load is assigned a time factor to decide its priority and then threshold criteria is calculated to find optimal time slot for each individual load. As it is a pure threshold policy. Therefore, this policy would be unfavorable when energy consumption and market spot pricing trends are dynamic. A similar work is proposed [5] to schedule residential loads in conjunction with on-site renewable energy and storage system. A mixed integer linear programming algorithm is used to solve cost minimization and comfort maximization problems. A slightly different work is presented [6] for real-time pricing demand response with fault-tolerant and flexible user enrolment to predict dynamic pricing. This scheme is relatively efficient in terms of less computational overhead and transmission delay. In [7], a two layered model for a hybrid energy system is proposed with a demand biding strategy. Before scheduling the load, a demand bidding based on Nash equilibrium theory is used to find the optimal pricing involving different stakeholder. Then, a coordinated multiagent framework is used to ensure the stability of this system with an event triggered mechanism. The work presented in [8] is devoted to modeling a locational marginal pricing based demand response using a monotonously decreasing linear function. In [9], a price based demand response program to schedule the residential load is presented and solved by using a decision support system. The load is first predicted and then scheduled based on market price to minimize overall cost. A novel pricing scheme considering residential demand response, renewable energy and power losses is presented for peak load alleviation [10]. All the generation and demand facilities actively collaborate in a distributed manner to find the best optimal price without affecting their objectives., where demand response DR allows the potential users to shift their consumption level or curtail some portion of the load in response to time-varying dynamic pricing such as time of use (TOU), real-time pricing (RTP), day-ahead pricing (DAP), and critical peak pricing (CPP) [11]. In [12], a multiagent based strategy to integrate the flexibility potential of industrial and residential demand is presented. A particular focus is on considering the cement and metal smelting industry, where residential and industrial demand is fulfilled through renewable and grid energy sources, respectively. In [13], a multiagent based load scheduling scheme is proposed, which utilizes optimal stopping theory to obtain the optimal scheduling instants. To optimally utilize the grid and renewable energy resources, a heuristic algorithm is used to solve the load scheduling problem [14]. Furthermore, to maximize user comfort in terms of scheduling delay, the load is modeled to prioritize comfort over cost and vice versa. To model the electricity prices, the authors first used a data mining approach to find the load consumption patterns from historical data using density based clustering with noise [15]. Then, a mixed integer nonlinear programming technique is applied to find electricity prices over the given time slots. To analyze the performance of this model, the online network enabled optimization system (NEOS) is used. The work presented in [16] is devoted to analyzing the advantages and disadvantages of active demand response programs in relation to residential load management. This work also analyzed the features of the energy system and highlights the key issues of decentralized energy resources. To reduce the peak load demand, the work presented in [17] is devoted to modeling the load and heating ventilation air-conditioning, particularly using demand response. In response to electricity prices and energy demand requirements, the dynamic demand response controller (DDRC) adjusts the control set points after every $15 \mathrm{~min}$ and shifts the load in case demand exceeds the predefined threshold. The proposed model is designed in the MATLAB/SIMULINK environment which is connected with the EnergyPlus model via a building control virtual 
test-bed. In [18], the authors proposed a renewable energy buying back scheme with a dynamic pricing strategy for energy efficiency in the smart grid. Here, the dynamic pricing is formulated as a convex optimization duel problem and a day-ahead tune dependent pricing in a distributed manner is proposed to ensure user privacy. This work is designed in such a way to provide the benefits to users and utility, dynamically. This is to note that the benefits offered by the DR program should not be underestimated due to their great impact on power system reliability and stability through its load management and optimization. However, the discussed benefits could be undermined due to the negligible impact of small power consumption loads on power system stability. Additionally, another drawback associated with the improper implementation of DR programs is the restriction of some high power rating loads being non-shiftable or non-interruptible [13]. On the other hand, EVs are becoming one of the potential candidates both in household and charging lots that draw a large amount of electric power, causing serious concerns from energy management perspectives [16]. There might be several reasons that exacerbate the utility's DR and create rebound peaks due to massive power that is drawn. Firstly, the aggregated charging loads of EVs can serve as a great resource of demand and capacity to obtain a noticeable impact on DR programs if there exists coordination among EV charging loads. Secondly, there should be greater flexibility on shifting and interruption offered by EV charging operators through the coordination with DR. This could manage EV charging load demand while fulfilling the key concerns of DR. These parameters are addressed and modeled in the work proposed in [19].

\section{Related Work}

Various price-based DR optimization techniques for optimally control residential loads have been reported in the literature. In [20], a new architecture based on technical building systems is presented, which is suitable for nearly zero energy building. To achieve this objective, the active load profiles of all the participants are obtained and then a building demand response program is used to model and manage the load for peak alleviation and flatten the load profile. These include model-based optimization methods [21], heuristic optimization techniques [22,23], where residential load is modeled with load classification [22] and customer preferences [23]. A stochastic optimization algorithms taking into consideration the inherent uncertainties in appliance scheduling time and electricity pricing [24]. To handle these uncertainties, a stochastic technique involving energy consumption adaptation variable and load consumption patterns is used. In [25], a probabilistic demand response program is proposed to model the load demand of residential sector. The main objective is to analyze the operational objectives used to balance the total cost. The stochastic optimization techniques provide the statistical results of the energy consumption of residential appliances. However, these algorithms are unable to guarantee an efficient DR policy from a day ahead perspective. On the other hand, model-based DR programs can guarantee the DR policy if the accurate load and patterns are available. However, a big challenge is how to get a correct estimate of the energy demand and usage patterns of residential loads. Because the energy consumption patterns cannot be fixed due to variable habits of energy consumers. In the third category, where home appliances are scheduled in response to dynamic prices using heuristic optimization methods. It is mentioned that optimal results in terms of cost and peak to average ratio reductions can be achieved with compromise on discomfort. Because, unlike electricity cost minimization, the comfort/social welfare maximization are two different objectives and cannot be achieved at the same time.

Moreover, the model-based DR schemes are developed based on simplified energy consumption models. In [26], the energy consumption of a residential house is modelled using simplified conduction heat transfer equations. In [27], the authors use a quasi-steady state approach for the estimation of a day-ahead electricity demand in the DR market. In [2], the authors adopt a model predictive control strategy that employs the model of building dynamics based on a thermal resistance-capacitance network. A low voltage 
residential load model based on price based DR has been proposed in [28]. In this work, high-resolution load models were developed by combing Monte Carlo Markov chain based bottom-up demand models, time-variant load model, shot water demand models and discrete state-space representation of thermal loads. Then, a price based DR program is modelled to control the working of all these loads in response to consumers. The model is useful for predicting the distributed impact of introducing dynamic pricing in the system. In [29], the authors consider the problems of considering dynamic pricing in the network. They have used a Stackelberg game approach to model the interactions between endusers and electricity producers. Then, a comprehensive characterization of the tradeoffs between consumer surplus and net profit is obtained. They have also analyzed the effects of renewable energy integration and distributed storage in the system. It is concluded that all benefits go to energy retailers when the capacity of renewable energy is small. These are the main conditions where renewable energy systems are used. In another similar work, the authors consider the problem of variable pricing and load consumption, where a utility or energy retailer acts as an agent between retailer and consumers [30], whereas variable pricing is somehow useful for the electricity retailer in stabilizing the electric network. However, its implementation seems difficult due to the lack of information about the consumers and associated uncertainties. Similarly, consumers are also more likely to face the difficulties in establishing their schedules for loads scheduling due to variable pricing. However, this problem can be tackled by using a reinforcement learning technique without background information of consumers and retailers. In [31], the authors propose a demand-side management (DSM) technique that takes into consideration user preferences. The authors identified a trade-off between cost reduction and comfort maximization and developed a Game theoretic-based algorithm to overcome this trade-off. In [32], the authors proposed a new pricing mechanism for low and high energy consumption users based on a time of use pricing policy. The price signal is further divided into different blocks and forecasted load is scheduled based on these prices to curtail the cost of end users. Here, an artificial neural network, due to its efficiency, is adopted to forecast the short term load in a day-ahead fashion. However, a few other algorithms to forecast the short and long term load are also being widely used by different authors [33]. In another similar approach, [34], the consumer's behaviours on each other's DSM decisions have been accounted for. Then a non-cooperative game strategy is used, where each user will decide whether to participate in the DR program or not. Here, to minimize the electricity cost is the main objective. While in traditional game-theoretic approaches, it is being considered that consumers are free in taking their decisions. In [35], the authors show that incoordination between consumer and electricity distributor may create the chances of high and rebound peaks leading to catastrophic behavior of the electric grid. In this regard, the authors propose a system-wide framework to coordinate the DR of end-users in a smart grid. The key objective of this framework is to provide monetary benefits, privacy and comfort to end-users.

\section{Motivation}

With population growth and advancements in information and communication technologies (ICTs), the power demand has also been increased. Consequently, scientists and researchers have been working to find new energy sources and energy management mechanisms to meet the growing power demand. In this regard, the SG vision has come to incorporate distributed and renewable energy resources, advance metering infrastructure (AMI) [36] and communication protocols, DR programs [20,21], and optimization techniques to efficiently manage the power demand. In accordance with this, the residential customers have been provided with the facility to curtail their load or reschedule the working slots in response to the DR programs and time-varying prices found in the literature [6-10]. Consequently, the utility obtained the benefits regarding of grid stability, while users can gain get the reduced bill as these pricing mechanisms are designed in such a way that the hourly price factor is calculated based on aggregated load con- 
sumed/demanded in a particular region which is operated under the same DSO. In almost all the techniques, DSM algorithms are used to schedule the load for energy management by taking into consideration the load demand, market clearing price, dynamic consumption trends and optimization algorithms. To achieve the effective outcomes of the DR based load scheduling programs, the end users are being offered various incentives in terms of bill reduction, uninterruptible supply of power, and so forth. However, the users are given the opportunity to decide whether they are willing to participate or not. Unlike the participating customers, the other customers would not be able to receive any benefit until they agreed to participate in DR programs by accepting terms and conditions. Furthermore, it is also understood that there could be multiple energy retailers being operated in some specific geographic region with dynamic and time varying price signals. The participating users have to manage their load according to the available price signal without knowing which other users are impacting on their benefits. For example, the high, medium and low energy consumers are always charged electricity bills according to the consumed load based on DR pricing policy. Here, the price signal would remain the same even if the customers are consuming low or high power over a given time period. Consequently, the those customers who have maintained a stable load profile and are consuming relatively less power are not getting the actual benefits or incentives in participating in DR and load scheduling programs. This is due to the discriminatory price signal being provided by the market retailer because it is difficult to provide a separate price signal to every user, which is a limitation of price based DR programs. Thus, by consideration of this problem and the underlying limitation of the DR program, this work proposes a new mechanism to provide some incentives to the customers maintaining a balanced load profile.

\section{Contribution}

This mechanism must possess the characteristics of a fair distribution of prices and incentives without any discrimination. By considering the aforementioned limitations and drawbacks, this paper further investigates the price based DR programs and introduces a novel mechanism for load management and price calculation with dynamic incentives. By taking into account the existing mechanisms (i.e., TOU, RTP) for calculating electricity prices, we have designed a new and slightly different method to calculate electricity prices and incentives based on load consumption and shifting patterns over given time period. For this purpose, the profiles of each user are obtained via smart meters using advanced metering infrastructure (AMI) [18] and are maintained just like the charging management system (CMS) in the electric vehicle scenario [28]. Then, by using the proposed mechanism, the price is calculated whether the user will get the incentives or overpriced signal, which is independent of each user and load. One of the unique and novel aspects of the proposed mechanism is that these incentives are non-discriminatory and independent from other users participating in a load scheduling based incentivise mechanism. These prices rather depend upon individual consumption trends and the RTP signal over the given time period. Hence, the proposed mechanism is designed to facilitate the users in reducing high and rebound peaks by incorporating the upper limit of energy consumption during the scheduling process. However, this may be inconvenient for those users who intend to use energy as per their defined schedules to improve the social welfare level. Consequently, the utility may receive extra benefits in terms of system stability through reliable energy generation and supply systems. Finally, the developed system model is tested and validated using the proposed algorithm which presents the idea of calculating dynamic incentives instead of static and predefined incentives.

\section{Characterizing DR}

As understood, electricity cannot be stored at large scale power systems due to physical limitations and constraints. Therefore, it is strongly recommended that the difference between electricity generation and consumption must be minimal. Moreover, the marginal cost of electricity in a day-ahead market is extremely dynamic due to variable 
and unexpected energy consumption patterns, whereas the electricity cost varies over the given period (i.e., $60 \mathrm{~min}, 30 \mathrm{~min}, 15 \mathrm{~min}$ ) of fixed intervals, and consumers receive retail electricity prices reflecting average energy generation trends including transmission and distribution losses. Therefore, the frequent disconnections between short term marginal and long term marginal costs can cause serious concerns regarding real-time energy generation and consumption. This is because consumers do not have the information about the marginal cost of supplied electricity. This results in an inefficient participation in DR programs. Consequently, the consumers receive little or no incentive for participating in DR programs. From the above-mentioned discussion, it can be concluded that a flat rate tariff encourages the consumers to overuse electricity during low pricing hours. As a result, electricity tariffs may have higher values due to excessive energy consumption during particular hours. Therefore, utilities have to fulfil energy demand by turning on extra generators leading to costly tariffs. In conclusion, inefficient utilization of DR programs and calculation of electricity tariffs in a day-ahead market may affect a consumer's objectives. As a response, consumers may be discouraged to adopt DR programs, which can create serious concerns regarding grid stability in the long term. So, it is desired to adopt some real-time mechanisms that could be feasible for both utilities and consumers, without disturbing/violating their objectives.

\section{System Model}

In this section, we describe the proposed system model used to calculate incentive based electricity prices. Let $i \in N$ denote the loads $l_{i}$ with time deadline denoted by $t \in T$ such that $t=1,2,3, \ldots T$ and $j \in M$ denotes index of users who are participating in load scheduling program and getting incentives. The energy demand of each load $i$ is denoted by $d_{l_{i}}(t)$. Let $\phi(t)$ denoted the global pricing policy (i.e., RTP) obtained from the electricity market via AMI. Let $\beta$ be the binary decision variable and represents the ON/OFF status of load. Each load has a predefined start $\tau_{i}^{s}$ and end time $\tau_{i}^{e}$, which lies within scheduling time interval $T$. Without losing generality, all customers have the equal opportunity to schedule their loads within the given time frame. Consequently, the cost-sensitive customers' $\mathbb{k}_{1}$ can get some incentives by shifting some load from on-peak to off-peak hours. In contrast, the customers $\mathbb{k}_{3}$ who are not willing to participate in DR programs may receive higher prices, which are calculated based on energy price and demand during critical hours. The details of $\mathbb{k}_{1}$ and $\mathbb{k}_{3}$ are given in the next section.

\subsection{Previous Model}

In traditional schemes being used to calculate energy consumption prices, where all users receive global prices irrespective of individualized demands, this may result in rebound peaks, that is, due to demand shifting from critical hours to off-peak hours $[1,37,38]$. In consequence, the non-homogeneous cost may be charged to customers, which participated in DSM programs. In addition, exposing end-users to the wholesale electricity market (i.e., real-time and day-ahead prices) has to lead the users to shift their load to a low pricing area, which eventually increases demand during this time span [38]. This would eventually lead to higher peaks. It is also demonstrated in [37] that this may create rebound peaks due to demand shifting, and hence, DSMs need to be studied in accordance with homogeneous and non-homogeneous energy users with the objective of peak shaving and fair price distribution among all types of consumers. Similarly, the work reported in [39] provided the concept of user aware price policies, which is based on individualized demand profiles obtained from smart grid communication network. Although the concept is novel, which helps with alleviating non-homogeneous price penalties on potential users. However, in reality, energy consumption has a dynamic trend which is based on customers' daily life activities. Eventually, this mechanism [39] may create homogeneous cost profiles for a given time. A general energy price is calculated using Equation (1).

$$
p_{i}=\sum_{i \in N} \sum_{t \in T}\left\{\left[\left(\beta \times \ell_{i}(t)\right) \times d_{\ell_{i}}(t)\right] \times \phi(t)\right\}, \forall i \in T, t \in T,
$$


where $\phi(t)$ is the real time electricity price obtained from a day-ahead market, $\beta$ is a binary decision variable which denotes, that is, ON/OFF states of connected loads $\ell_{i}(t)$ during time $t$, and is expressed as;

$$
\beta= \begin{cases}1 ; & \text { If load is ON } \\ 0 ; & \text { If load is OFF. }\end{cases}
$$

As the utility pricing model is based on some specified time interval, which is considered $24 \mathrm{~h}$ equally divided on all time slots. Therefore, $t_{s}$ and $t_{e}$ are used to denote start and end time intervals of $\ell_{i}$ over the given price $\phi(t)$, respectively, whereas the $\phi$ is obtained from the electricity retail market, which is changed due to load trends and consumer demand over the given period $[t \in T]$, while $\tau_{i}$ denotes utility cycles (i.e., number of time slots) of all loads subject to $\left(\beta \ell_{i}(t)\right)$. The following sections discuss different cases related to electricity price calculation methods.

\subsection{Demand Aware Prices (Case-1)}

To cater for the aforementioned uncertainty, the proposed work provides a mathematical mechanism to calculate electricity prices on the basis of energy consumption profiles of all users/units which are obtained from smart grid communication network. For this purpose, there need to be community networks to handle individual price profiles of all users in accordance with the individualized energy consumption trends. The electricity price $\left(p_{i}^{\prime}\right)$ is calculated by using the proposed mechanism, which depends on the load consumption of each customer, and is expressed through Equation (3):

$$
p_{i}^{\prime}(t)=\sum_{i \in N} \sum_{t \in T}\left\{\left[\left(\beta \times \ell_{i}(t)\right) \times d_{\ell_{i}}(t)\right] \times \phi^{\prime}(t)\right\}, \forall i \in T, t \in T,
$$

where $\phi^{\prime}(t)$ denotes homogeneous price policies which further depends on $\mathbb{k}_{1}$ variable denoting the actual electricity price each customer will be charged, giving the variation in energy consumption of $\ell_{i}$ over give time $t$. The $\mathbb{k}_{1}$ can be calculated on the bases of $\ell_{i}(t)$ and $\phi(t)$ and expressed in Equation (4):

$$
\begin{gathered}
\mathbb{k}_{1}(t)=\sum_{i \in N} \sum_{t \in T}\left\{\left[\left(\left(\beta \times \ell_{i}(t)\right) \times d_{\ell_{i}}(t)\right) \times \phi(t)\right] \times\left(d_{i}\right)^{-1 / 2}\right\}, \forall i \in T, t \in T, \\
p_{i}^{\prime}(t)=\sum_{i \in N} \sum_{t \in T}\left\{\left[\left(\beta \times \ell_{i}(t)\right) \times d_{\ell_{i}}(t)\right] \times \mathbb{k}_{1}(t)\right\}, \forall i \in T, t \in T .
\end{gathered}
$$

Equation (5) denotes the electricity price $\left(p_{i}^{\prime}\right)$ in the proposed case and the cost minimization objective function can be described through Equation (6):

$$
\begin{gathered}
\min \sum_{i \in N} \sum_{t \in T} \Phi_{i}(t) \\
\text { s.t }: \forall i \in N: \Phi_{i}=\left(\sum_{i \in N} \sum_{t \in T} p_{i}^{\prime}(t) .\right)
\end{gathered}
$$

\subsection{An Incentive Based Price Overview (Case-2)}

In this case, we first identify the total users consuming high power, contributing towards a high electricity tariff. Eventually, the other users who have a balanced power consumption profile may be affected in terms of bearing high prices. This section aims to devise a mechanism to fairly design customized price profiles for all users. For this purpose, the pricing tariff for the customers consuming high power needs to be calculated, which would be used in calculating incentives for the customers showing balanced power consumption trends. After calculating incentives and penalties for respective customers, we will then compare the cost values of both traditional and proposed mechanisms for 
validation purpose. Using Equation (8), we can identify the consumers who have drawn more power leading to adding more to the electricity price being provided.

$$
p_{i}^{\prime}(t)-p_{i}(t)=0, \forall i \in T, t \in T .
$$

Now, we can design the customized electricity tariff for high consumption users. Meanwhile, we also calculate the incentives to those customers who willingly maintain stable load profile to seek incentives from utility.

\subsection{An Incentive Based Price Calculation}

This section discusses the incentive-based price policies designed for those customers who participated in the energy management process $j \in M$ to reduce peak load demand. In response, the participating customers have been given incentives in the form of a bill reduction. In contrast, the customers who do not want to compromise on their comfort level and have not yet participated in energy management programs are charged prices in accordance with load demand and non-discriminatory penalties. These penalties have been calculated to preserve utility revenue. Initially, we have to calculate the electricity cost of two types of customers: (i) the customers with surplus electricity cost being charged after participation in the energy management (EM) process; and (ii) the customers with reduced electricity costs after participation in the EM process. For this purpose, we first calculate the reduced cost as expected by the $M$ customers, which can be calculated through Equation (9):

$$
\begin{array}{r}
p_{i}^{\prime}(t)=\sum_{j \in M} \sum_{i \in N} \sum_{t \in T}\left\{\left[\left(\beta \times \ell_{i, j}(t)\right) \times d_{\ell_{i, j}}(t)\right] \times \mathbb{k}_{2}(t)\right\}, \forall i \in, j \in J, T, t \in T \\
\mathbb{k}_{2}(t)=\sum_{i \in N} \sum_{j \in M} \sum_{t \in T}\left\{\left[\left(\left(\beta \times \ell_{i, j}(t)\right) \times d_{\ell_{i, j}}(t)\right) \phi^{\prime}(t)\right] \times\left(d_{i, j}\right)^{-1 / 2}\right\}, \forall i \in T, t \in T,
\end{array}
$$

where Equation (10) denotes the fraction by which the customers $j$ consumes more energy, and $\phi^{\prime}(t)$ is the price, which is expected by customers $j$ in order to reduce their electricity cost. The actual electricity price $p_{i}^{\prime \prime}(t)$ which is obtained after load scheduling is expressed through Equation (11):

$$
p_{i}^{\prime \prime}(t)=\sum_{i \in N} \sum_{j \in M} \sum_{t \in T}\left\{\left[\left(\beta \times \ell_{i, j}(t)\right) \times d_{\ell_{i, j}}(t)\right] \times \phi^{\prime \prime}(t)\right\}, \forall i \in T, j \in M, t \in T,
$$

where $\phi^{\prime \prime}(t)$ depicts the electricity price being charged to $j$ customers. Now, we can calculate the surplus electricity price being charged to $j$ customers, based on which the comfort cost is calculated for $i$ consumers. Once this cost is calculated, the extra cost charged to $j$ customers would be adjusted in order to provide incentives in the form of electricity bill reduction. The total amount of electricity cost for $i$ can be calculated through Equation (12):

$$
\overline{p_{j}}(t)=\sum_{i \in N} \sum_{j \in M} \sum_{t \in T}\left\{\left[\left(\left(\beta \times \ell_{i, j}(t)\right) \times d_{\ell_{i, j}}(t) \times\left(\mathbb{k}_{1}(t)+\alpha\right)\right)\right] \times\left(d_{i, j}\right)^{-1 / 2}\right\}, \forall i \in N, j \in M, t \in T,
$$

where $\overline{p_{i}}$ denotes the actual price charged to $M$ customers and $\alpha=\mathbb{k}_{2}-\mathbb{k}_{3}$ is the approximate cost difference between scheduled and unscheduled cases. $\mathbb{k}_{3}$ can be through Equation (13):

$$
\mathbb{k}_{3}(t)=\sum_{i \in N} \sum_{j \in M} \sum_{t \in T}\left\{\left[\left(\beta \times \ell_{i, j}(t)\right) \times d_{\ell_{i, j}}(t) \times \phi^{\prime}(t)\right] \times\left(d_{i, j}\right)^{-1 / 2}\right\}, \forall i \in N, j \in M, t \in T .
$$

Now, the total energy consumption cost $\hat{\Phi}_{i}(t)$ over time $t$ is calculated by using Equation (14); 


$$
\begin{aligned}
& \hat{\Phi_{i, j}}(t)=\left\{\overline{p_{i}} \sum_{i \in N} \sum_{t \in T}(t)+\left[\sum_{i \in N} \sum_{j \in M} \sum_{t \in T}\left\{\left[\left(\beta \times \ell_{i, j}(t)\right) \times d_{\ell_{i, j}}(t)\right] \times \mathbb{k}_{3}(t)\right\} \times\left(d_{i, j}\right)^{-1 / 2}\right] \times \sum_{i \in N} \sum_{j \in M} \sum_{t \in T} d_{i, j}(t)\right\}, \\
& \forall i \in N, j \in M, t \in T .
\end{aligned}
$$

Now, we can calculate incentives for $M$ customers who took part in the energy management program. Let $\mathbb{E}_{\bar{p}_{i}}$ denote the monetary incentives given to those customers who have participated in the energy management process to flatten the peak demand during critical hours. From the above Equations (1)-(12), we can calculate $\mathbb{E}_{\bar{p}_{i}}$ as given below;

$$
\mathbb{E}_{\overline{p_{i}}}=\sum_{i \in N} \sum_{j \in M} \sum_{t \in T}\left[\hat{\Phi_{i, j}}(t)-\left\{\mathbb{k}_{3}(t) \times\left(\left(\beta \times \ell_{i, j}(t)\right) \times d_{\ell_{i, j}}(t)\right) \times \phi^{\prime}(t)\right\}\right], \forall i \in N, j \in M, t \in T .
$$

Now, the final objective function Equation (16) which is the incentive maximization and subject to the constraints of Equations (17)-(20) is written as:

$$
\begin{aligned}
& \min \sum_{i \in N} \sum_{j \in M} \sum_{t \in T}\left(\mathbb{E}_{\bar{p}_{i, j}}(t)\right) \\
& \mathbb{E}_{\bar{p}_{i}} \leq\left(d_{\ell_{i}} \times \phi\right): \forall i \in N .
\end{aligned}
$$

Equation (16) refers to the cost minimization objective function and Equation (17) denotes that the total cost obtained from the proposed mechanism should always be less than the traditional mechanism. Otherwise, the proposed mechanism is not an optimal one.

$$
\begin{gathered}
\hat{\Phi}_{i}=u_{r}: \forall i \in N \\
p_{\ell_{i, j}^{u n}}=p_{d_{i, j}^{s c h}}: \forall i \in N .
\end{gathered}
$$

Equation (18) shows the total cost of load consumed, which should be equal to the cost/utility revenue. Otherwise, the mismatch could ultimately affect the objectives. Equation (19) denotes that the unscheduled and scheduled load before and after the scheduling process must be equal. Otherwise, the proposed mechanism could be based on energy conservation/reduction instead of load scheduling or management.

$$
\begin{gathered}
\overline{p_{d_{i, j}}}<p_{d_{i, j}}<\underline{p_{d_{i, j}}}: \forall i \in N \\
\ell_{i} \leq \beta \times \ell_{u t i}(t): \forall i \in N, t \in T .
\end{gathered}
$$

Equation (20) shows the upper and lower limits on the load demand and Equation (21) shows that the load demand must not exceed the utility capacity.

\section{Proposed Algorithm}

In the proposed work, we used GA to solve the incentive-based load scheduling problem. Although, the consideration of heuristic algorithms is gaining popularity due to their ability to obtain the best optimal results even when other mathematical algorithms fail due to the diverse nature of control parameters and inherent complexity while designing or formulating the underlying problem, mathematically. That is why we have considered and used GA due to its ability to obtain global optimal results in all situations with a high convergence rate. Moreover, the crossover and mutation operators further allow obtaining the best results even if some random parameters affect the performance of the results. Generally, convergence is one of the important parameters when dealing with successive series of experiments in iterative methods [36,40]. Generally, the GA works by considering some initial parameters with random initial population, fitness function, and values of other control parameters. Initially, the objective/cost function is evaluated as per 
given control values such as cost, incentives and delay in the proposed work. Once the fitness is calculated and the best results are obtained, the algorithm stops by following some predefined criteria or based on an initial number of iterations. Then, the best optimal results are saved and crossover and mutation operators are applied to evaluate the fitness function based on a new population. The probabilities of crossover $P_{c}$ and $P_{m}$ are set in such a way to obtained global optimal results. However, to avoid premature convergence, we have compared the best convergence results with the newly obtained results in each iteration and used a Sigma scaling factor so that premature results should be discarded. We have also tested the convergence against different control values to assess the performance under the proposed model. It is also worth mentioning here that the performance of any optimization algorithm also depends on the well behaved functional form of a developed mathematical model. Otherwise, the optimal results may take more time or face complexities in achieving them. Table 1 provides the loads with demand and working hours, while the Table 2 gives the values of control variables used to find the optimal scheduling patterns and incentives. Max. generation is selected as 800 so as to have the sufficient space for optimal results. The population size is chosen as 400 in order to avoid the solution being premature. The $P_{c}$ and $P_{m}$ values are selected and set after monitoring the convergence results. We observed through experiments that these values are the most suitable for fast convergence. The Algorithm 1 explains the working steps involved in getting incentives based price profiles. In step-4, the initial random population is generated which is evaluated in step-5 using Equation (7). Step-6 is involved in calculating the power using RTP and proposed mechanisms. Equations (9), (11), (12), (14) and (15) are involved for the calculation. Steps 1-8 show that the algorithm moves to the next step if an optimal solution is obtained. Otherwise, the control will move to step-1 for new calculation and evaluation. Steps 13-14 compare the optimal solutions obtained from Equation (16) and Equation (6). After saving the results, the crossover and mutation operators are applied and the control moved to the step-1. The same process repeats over a $24 \mathrm{~h}$ time period.

Table 1. Load consumption and duty cycle requirements.

\begin{tabular}{ccc}
\hline$l_{\boldsymbol{i}}$ & Working Hours & $\boldsymbol{d}_{\boldsymbol{l}_{\boldsymbol{i}}}(\mathbf{k W})$ \\
\hline$l_{1}$ & 20 & 2.5 \\
$l_{2}$ & 24 & 3 \\
$l_{3}$ & 5 & 2 \\
$l_{4}$ & 7 & 2.5 \\
$l_{5}$ & 8 & 3.5 \\
$l_{6}$ & 8 & 3 \\
\hline
\end{tabular}

Table 2. Control parameters of GA [36,40].

\begin{tabular}{cc}
\hline Parameters & Values \\
\hline Number of loads & 6 \\
Number of users & 3 \\
Max. generation & 800 \\
Population size & 400 \\
Probability of crossover & 0.9 \\
Probability of mutation & 0.003 \\
\hline
\end{tabular}




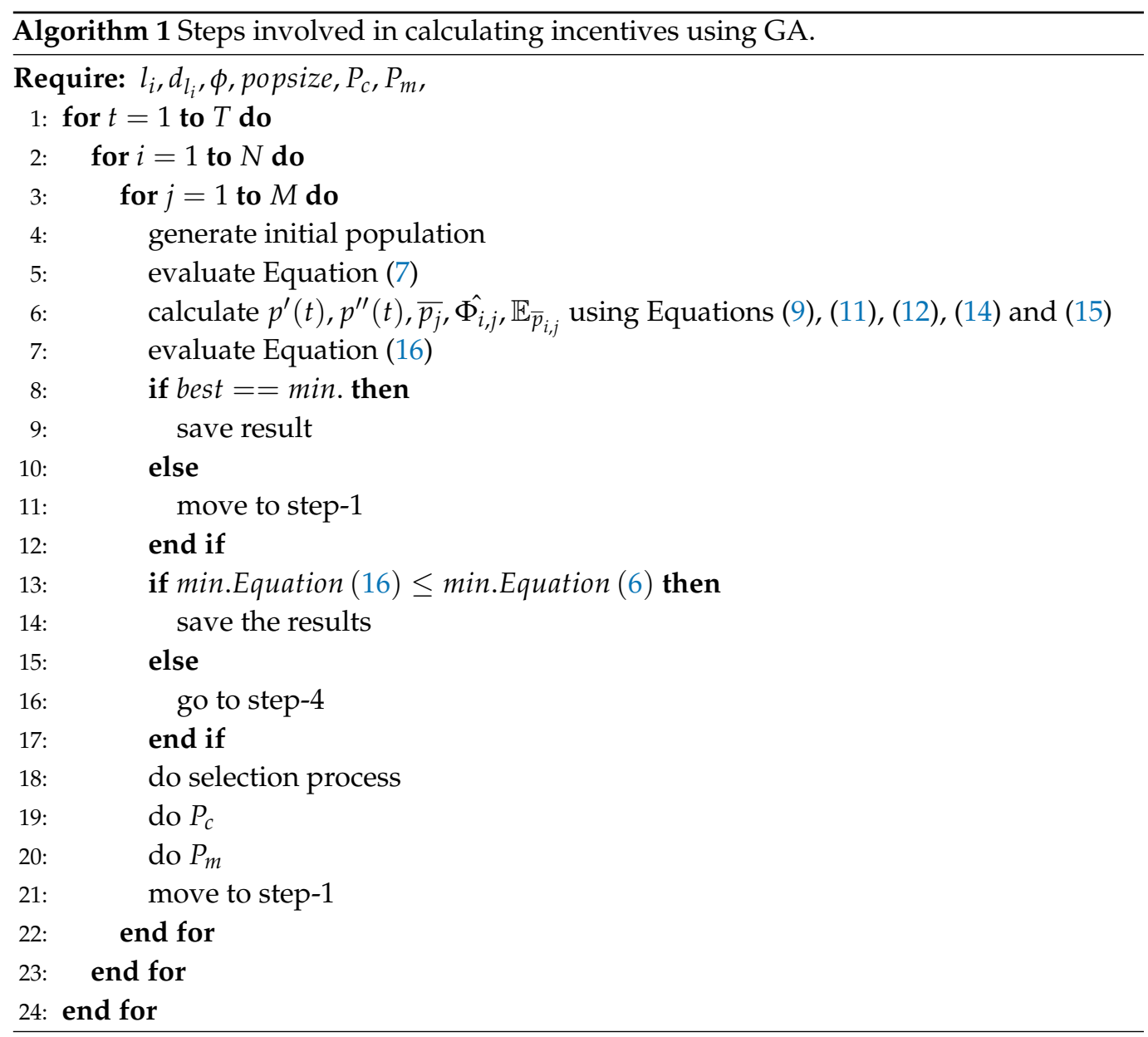

\section{Results and Discussion}

Figure 1 shows the electricity price signal used in the proposed work. Unlike other pricing signals being widely used in the literature and real-time works such as RTP, TOU, DAP, CPP, the proposed price signal is dynamic with changing values at every instant of time. This price signal is considered to deeply analyze the realistic cost and incentive profiles of all the consumers instead of developing the load scheduling techniques of algorithms based on DAP, which is known in advance to the users and energy management controllers. Furthermore, these price signals elucidate the realistic changing behaviours of price with respect to load. Figure 2 gives the convergence profile of GA based on the control and load scheduling parameters. It is clear from the figure that GA converges within 400 iterations and results with the best optimal cost in each iteration are obtained. Figure 3 give and comparison of the price profiles between the RTP signal and the proposed pricing signals being calculated using proposed incentive based model. It can be seen from this figure that the proposed price profiles differ from RTP, showing that the proposed mechanism calculates the price signal based on load demand, scheduling capacity and incentives, respectively. Figure 1 shows a comparison of different patterns of price profiles using RTP and proposed price (PP). From first sight, it is very clearly visible that the price profiles using PP has variations when compared with RTP obtained from a day-ahead market. These variations are due to various incentives provided to different consumers based on load shifting and taking part in the load scheduling mechanism. For example, if any user is willingly participating in load scheduling and maintain a consumption level under the upper threshold limit as compared to the other consumers, he gets incentives in electricity tariffs of that particular hour, instead of for the whole day. Similarly, if any user is consuming relatively more power and exceeding the upper threshold limit, then s/he has to be charged the extra price due to creating trouble for the electricity retailer of the producer. 
This is because the electricity producer or distributor has to maintain a balance between generation and demand. Figure 4 provides the energy consumption profiles of all the units using RTP. $\mathrm{t}$ can be seen that scheduled load profiles show remarkable trends reflecting the scheduling capability of the proposed mechanism based on GA algorithm, which is in terms of load scheduling and peak management as well. Similarly, Figure 5 shows the cost profiles of all the units using RTP. These variations provide an overview of the scheduling mechanism designed to lower the overall cost and providing incentives to the users. It is also seen from the profiles that scheduled cost is lower than unscheduled cost. Here, the maximum peak is around $350 \$$ and the minimum peak is around $20 \$$. In contrast, Figure 6 provides the cost profiles of all the units using PP. Here, we can see that the maximum peak is around $370 \$$ and the minimum peak is around $10 \$$. It reflects that those customers who are willingly participating in the proposed scheduling mechanism are getting the incentives in terms of reduced cost. On the other hand, the other customers who are not interested in participating in load management programs are getting a comparatively higher price and cost.

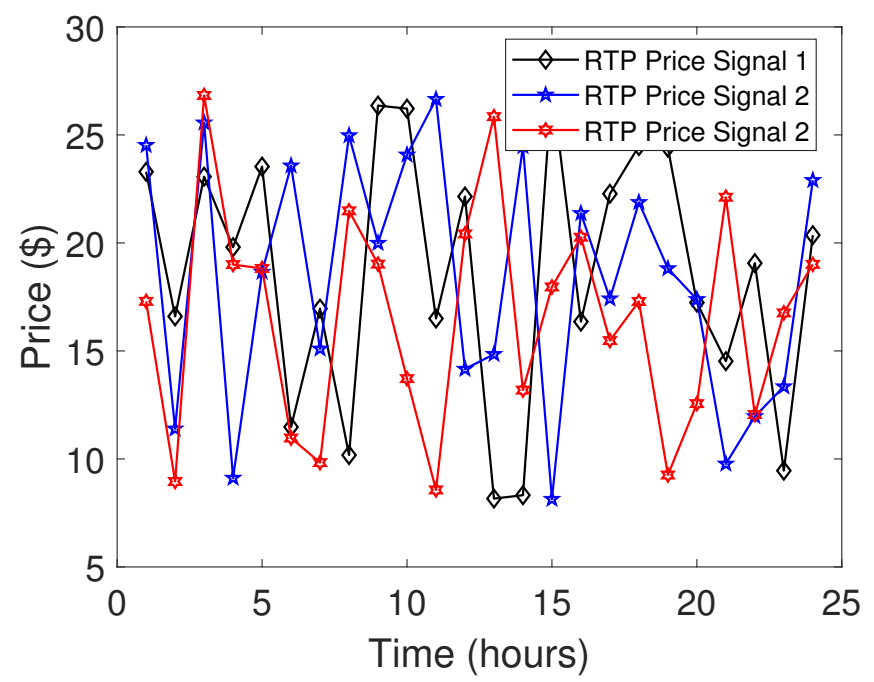

Figure 1. Real time electricity price signals over time $t$.

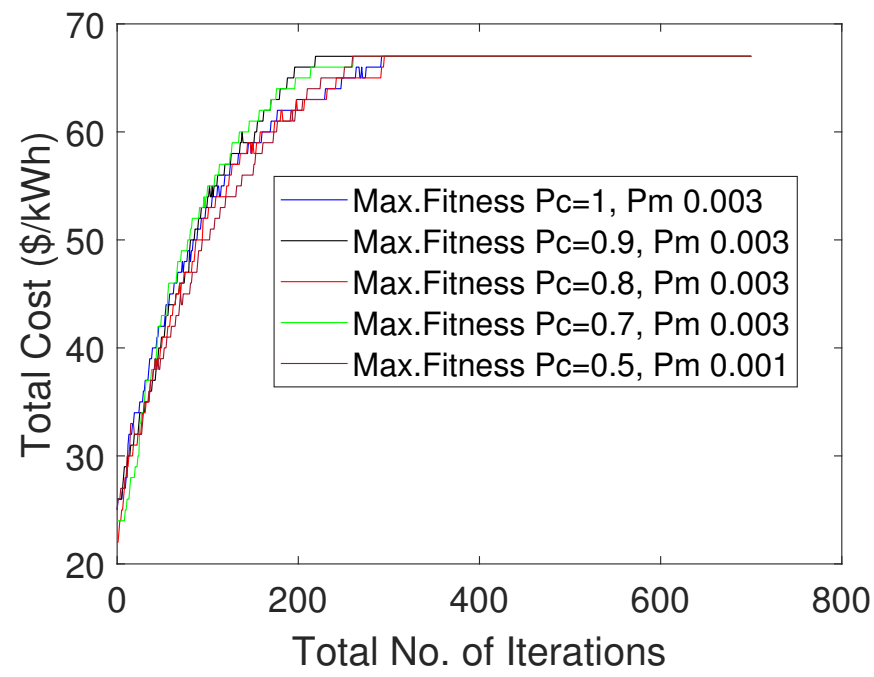

Figure 2. A comparison between total cost and fitness value over different values of control parameters. 


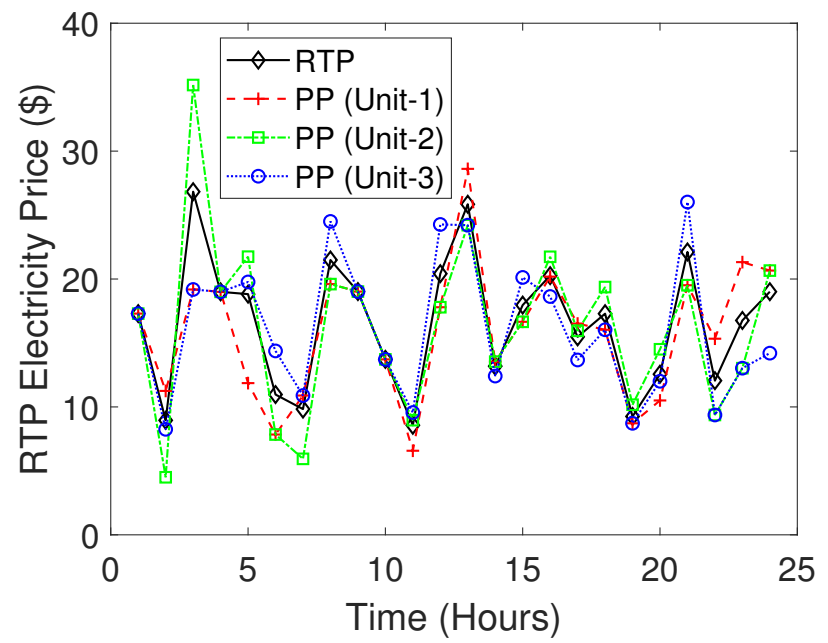

Figure 3. A comparison between real time electricity price and the proposed method for different units over time $t$.

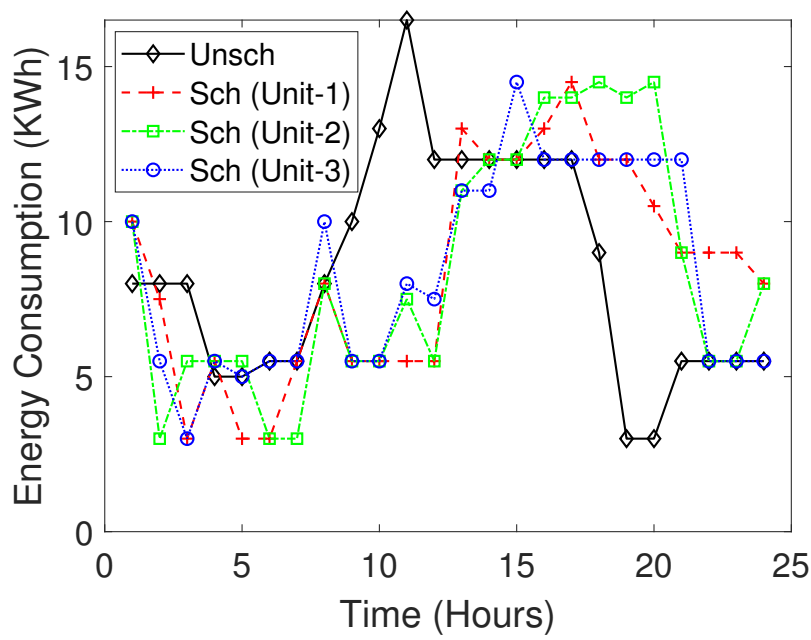

Figure 4. A comparison of energy consumption using real time electricity and proposed price signals for different units over time $t$.

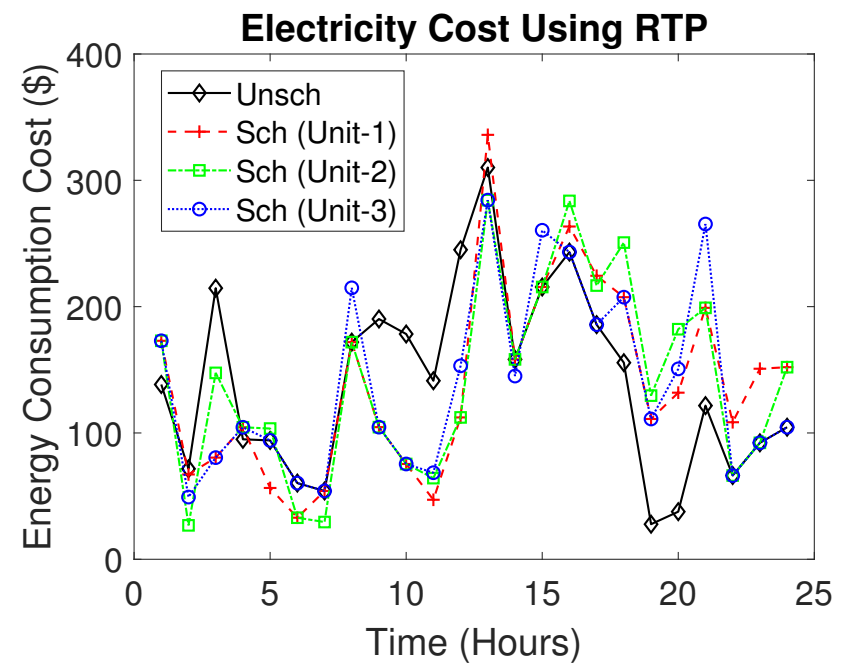

Figure 5. Electricity cost incurred for different units over time $t$ using real time price signal. 


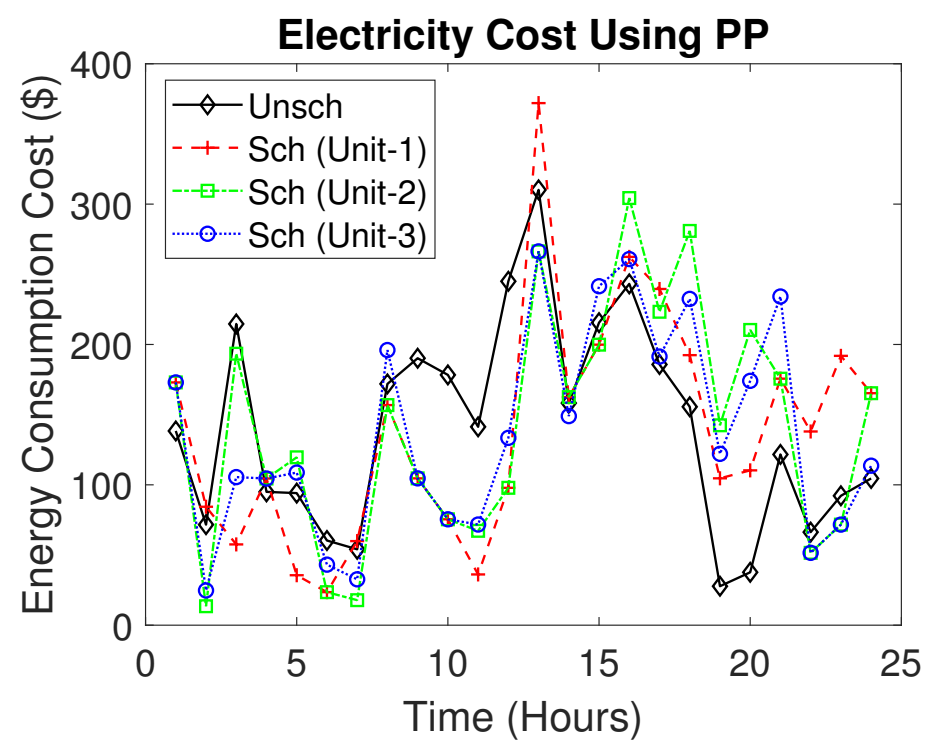

Figure 6. Electricity cost incurred for different units over time $t$ using proposed method.

\section{Conclusions}

Price based DR programs through AMI are designed to encourage users to participate in load scheduling processes to balance generation and demand capacity without considering costlier generation. However, these programs faced difficulties in attracting a large number of customers to participate. This is due to the inherent limitations caused by its discriminatory nature if applied in different geographical regions being operated under the same distributed system operator. To handle this limitation, this work has proposed an incentive-based load scheduling mechanism using a real-time pricing policy to facilitate energy retailers' and end-users' particularity. The incentives, in terms of dynamic pricing profiles using load consumption trends, are calculated. However, as these incentives are calculated based on the dynamic price profiles of different customers, we first obtained these profiles based on load consumption trends. In response, each customer received a different price signal, which is dynamically changed with load consumption reflecting incentives or an overpriced tariff. To analyze the impact of the proposed mechanism, we provided a system model to calculate these prices and incentives and formulate an objective function. Then, a well behaved functional form of optimization function is solved by using a heuristic based GA and obtained the energy and load consumption profiles. The results are then compared with the unscheduled profiles without incentives. It is clear from the results that participating customers are able to get dynamic pricing signal based incentives without affecting the electricity bills of other customers. In the future, we intend to extend and implement this model in designing electricity prices in peer-to-peer energy transactions using blockchain technology.

Author Contributions: T.A., M.B.R. and M. Awais did the mathematical modelling and implementation. T.A., A.H.M. check and verify the results. T.A., A.H.M. and M.B.R. supervise the project. All authors have read and agreed to the published version of the manuscript.

Funding: This project was funded by Deanship of Scientific Research (DSR) at King Abdulaziz University, Jeddah, under grant number (RG-13-135-41). The authors therefore acknowledge with thanks DSR for their technical and financial support. This project has also received funding from the European Union Horizon 2020 research and innovation programme under the Marie Sklodowska-Curie grant agreement No 754382, GOT ENERGY TALENT. The content of this [report/study/article/publication] does not reflect the official opinion of the European Union. Responsibility for the information and views expressed herein lies entirely with the author(s).

Institutional Review Board Statement: Not applicable.

Informed Consent Statement: Not applicable. 
Data Availability Statement: No new data were created or analysed in this study. Data sharing is not applicable to this article.

Acknowledgments: The authors thank the referees for careful reading and useful comments that helped to improve the paper.

Conflicts of Interest: The authors declare no conflict of interest.

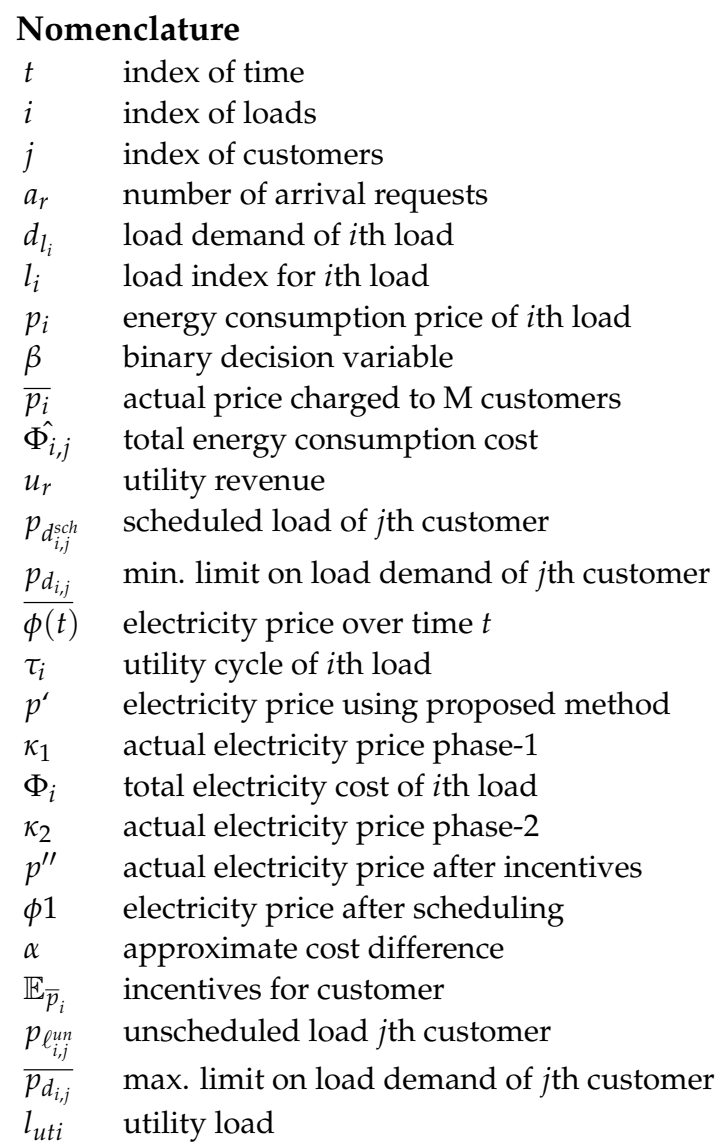

\section{References}

1. Baimel, D.; Tapuchi, S.; Baimel, N. Smart grid communication technologies-overview, research challenges and opportunities. In Proceedings of the 2016 International Symposium on Power Electronics, Electrical Drives, Automation and Motion (SPEEDAM), Capri, Italy, 22-24 June 2016; pp. 116-120. [CrossRef]

2. Siroky, J.; Oldewurtel, F.; Cigler, J.; Privara, S. Experimental analysis of model predictive control for an energy efficient building heating system. Appl. Energy 2011, 88, 3079-3087. [CrossRef]

3. Konda, S.R.; Mukkapati, B.; Panwar, L.K.; Panigrahi, B.K.; Kumar, R. Dynamic Energy Balancing Cost Model for Day Ahead Markets with Uncertain Wind Energy and Generation Contingency under Demand Response. IEEE Trans. Ind. Appl. 2018, 54, 4908-4916. [CrossRef]

4. $\quad$ Peizhong, Y.; Xihua, D.; Abiodun, I.; Chi, Z.; Shufang, L. Real-time oppertunistic scheduling for residential demand response. IEEE Trans. Smart Grid 2013, 4, 174-227.

5. Aslam, S.; Khalid, A.; Javaid, N. Towards efficient energy management in smart grids considering microgrids with day-ahead energy forecasting. Electr. Power Syst. Res. 2020, 182, 106232. [CrossRef]

6. Xue, K.; Yang, Q.; Li, S.; Wei, D.S.L.; Peng, M.; Menmon, I.; Hong, P. PPSO: A Privacy-Preserving Service Outsourcing Scheme for Real-Time Pricing Demand Response in Smart Grid. IEEE Internet Things J. 2019. [CrossRef]

7. Zhang, H.; Yue, D.; Dou, C.; Li, K.; Xie, X. Event-Triggered Multiagent Optimization for Two-Layered Model of Hybrid Energy System With Price Bidding-Based Demand Response. IEEE Trans. Cybern. 2021. [CrossRef] [PubMed]

8. Ding, T.; Qu, M.; Amjady, N.; Wang, F.; Bo, R.; Shahidehpour, M. Tracking Equilibrium Point under Real-time Price-Based Residential Demand Response. IEEE Trans. Smart Grid 2020. [CrossRef]

9. Ozturk, Y.; Senthilkumar, D.; Kumar, S.; Lee, G. An intelligent home energy management system to improve demand response. IEEE Trans. Smart Grid 2013, 4, 694-701. [CrossRef] 
10. Nguyen, D.H.; Narikiyo, T.; Kawanishi, M. Optimal Demand Response and Real-Time Pricing by a Sequential Distributed Consensus-Based ADMM Approach. IEEE Trans. Smart Grid 2018, 9, 4964-4974. [CrossRef]

11. Gholian, A.; Mohsenian-Rad, H.; Hua, Y. Optimal Industrial Load Control in Smart Grid. IEEE Trans. Smart Grid 2016, 7, 2305-2316. [CrossRef]

12. Golmohamadi, H.; Keypour, R.; Bak-Jensen, B.; Pillai, J.S.R. A multi-agent based optimization of residential and industrial demand response aggregators. Int. J. Electr. Power Energy Syst. 2019, 107, 472-485. [CrossRef]

13. Rasheed, M.B.; Javaid, N.; Malik, M.S.A.; Asif, M.; Hanif, M.K.; Chaudary, M.H. Intelligent Multi-Agent Based Multilayered Control System for Opportunistic Load Scheduling in Smart Buildings. IEEE Access 2019, 7, 23990-24006. [CrossRef]

14. Asgher, U.; Rasheed, M.B.; Al-Sumaiti, A.; Rahman, A.; Ali, I.; Alzaidi, A.; Alamri, A. Smart Energy Optimization Using Heuristic Algorithm in Smart Grid with Integration of Solar Energy Sources. Energies 2018, 11, 3494. [CrossRef]

15. Yang, J.; Zhao, J.; Wen, F.; Dong, Z. A Model of Customizing Electricity Retail Prices Based on Load Profile Clustering Analysis. IEEE Trans. Smart Grid 2018. [CrossRef]

16. Darby, S.J. Load management at home: Advantages and drawbacks of some active demand side options. J. Power Energy 2012, 227, 9-17. [CrossRef]

17. Yoon, J.H.; Baldick, R.; Novoselac, A. Dynamic demand response controller based on rea-time retail price for residential buildings. IEEE Trans. Smart Grid 2014, 5, 121-129. [CrossRef]

18. Chiu, T.C.; Shih, Y.Y.; Pang, A.C.; Pai, C.W. Optimized Day-Ahead Pricing with Renewable Energy Demand-Side Management for Smart Grids. IEEE Internet Things J. 2017, 4, 374-383. [CrossRef]

19. Ridoy, D.; Wang, Y.; Putrus, G.; Kotter, R.; Marzband, M.; Herteleer, B.; Warmerdam, J. Multi-objective techno-economicenvironmental optimisation of electric vehicle for energy services. Appl. Energy 2020, 257, 113965.

20. Martirano, L.; Habib, E.; Parise, G. Demand Side Management in Microgrids for Load Control in Nearly Zero Energy Buildings. IEEE Trans. Ind. Appl. 2017, 53, 1769-1779. [CrossRef]

21. Adika, C.O.; Wang, L. Demand-side bidding strategy for residential energy management in a smart grid environment. IEEE Trans. Smart Grid 2014, 5, 172-1733. [CrossRef]

22. Rasheed, M.B.; Javaid, N.; Ahmad, A.; Khan, Z.A.; Qasim, U.; Alrajeh, N. An efficient power scheduling scheme for residential load management in smart homes. Appl. Sci. 2015, 5, 1134-1163. [CrossRef]

23. Rasheed, M.B.; Javaid, N.; Ahmad, A.; Jamil, M.; Khan, Z.A.; Qasim, U.; Alrajeh, N. Energy optimization in smart homes using customer preference and dynamic pricing. Energies 2016, 9, 593. [CrossRef]

24. Chen, X.; Wei, T.; Hu, S. Uncertainty-aware household appliance scheduling considering dynamic electricity pricing in smart home. IEEE Trans. Smart Grid 2013, 4, 932-941. [CrossRef]

25. Harsha, P.; Sharma, M.; Natarajan, R.; Ghosh, S. A framework for the analysis of probabilistic demand response schemes. IEEE Trans. Smart Grid 2013, 4, 2274-2284. [CrossRef]

26. Black, J.W. Integrating Demand into the U.S. Electric Power System: Technical, Economic, and Regulatory Frameworks for Responsive Load. Ph.D. Dissertation, Engineering Systems Division, Massachusetts Institute of Technology: Cambridge, MA, USA, 2005.

27. Zhou, Z.; Zhao, F.; Wang, J. Agent-based electricity market simulation with demand response from commercial buildings. IEEE Trans. Smart Grid 2011, 2, 580-588. [CrossRef]

28. McKenna, K.; Keane, A. Residential load modeling of price-based demand response for network impact studies. In Proceedings of the 2016 IEEE Power and Energy Society General Meeting (PESGM), Boston, MA, USA, 17-21 July 2016; p. 1. [CrossRef]

29. Jia, L.; Tong, L. Dynamic Pricing and Distributed Energy Management for Demand Response. IEEE Trans. Smart Grid 2016, 7, 1128-1136. [CrossRef]

30. Kim, B.G.; Zhang, Y.; Schaar, M.v.; Lee, J.W. Dynamic Pricing and Energy Consumption Scheduling With Reinforcement Learning. IEEE Trans. Smart Grid 2016, 7, 2187-2198. [CrossRef]

31. Yaagoubi, N.; Mouftah, H.T. User-Aware Game Theoretic Approach for Demand Management. IEEE Trans. Smart Grid 2015, 6, 716-725. [CrossRef]

32. Aurangzeb, K.; Aslam, S.; Mohsin, S.M.; Alhussein, M. A Fair Pricing Mechanism in Smart Grids for Low Energy Consumption Users. IEEE Access 2021, 9, 22035-22044. [CrossRef]

33. Aslam, S.; Herodotou, H.; Mohsin, S.M.; Javaid, N.; Ashraf, N.; Aslam, S. A survey on deep learning methods for power load and renewable energy forecasting in smart microgrids. Renew. Sustain. Energy Rev. 2021, 144, 110992. [CrossRef]

34. Wang, Y.; Saad, W.; Mandayam, N.B.; Poor, H.V. Load Shifting in the Smart Grid: To Participate or Not? IEEE Trans. Smart Grid 2016, 7, 2604-2614. [CrossRef]

35. Safdarian, A.; Fotuhi-Firuzabad, M.; Lehtonen, M. Optimal Residential Load Management in Smart Grids: A Decentralized Framework. IEEE Trans. Smart Grid 2016, 7, 1836-1845. [CrossRef]

36. Katoch, S.; Chauhan, S.S.; Kumar, V. A review on genetic algorithm: Past, present, and future. Multimed Tools Appl. 2021, 80, 8091-8126. [CrossRef] [PubMed]

37. Li, Y.; Ng, B.L.; Trayer, M.; Liu, L. Automated residential demand response: Algorithmic implications of pricing models. IEEE Trans. Smart Grid 2012, 3, 1712-1721. [CrossRef]

38. Zhao, Z.; Lee, W.C.; Shin, Y.; Song, K.-B. An optimal power scheduling method for demand response in home energy management system. IEEE Trans. Smart Grid 2013, 4, 1391-1400. [CrossRef] 
39. Al-Rubaye, S.; Al-Dulaimi, A.; Mumtaz, S.; Rodriguez, J. Dynamic Pricing Mechanism in Smart Grid Communications Is Shaping Up. IEEE Commun. Lett. 2018, 22, 1350-1353. [CrossRef]

40. Houck, C.R.; Joines, J.; Kay, M.G. A genetic algorithm for function optimization: A Matlab implementation. Ncsu-ie tr 1995, 95, 1-10. 